\title{
The Importance of Quality in Ventilation-Perfusion Imaging
}

\author{
April Mann, CNMT, NCT, RT(N), FSNMMI-TS ${ }^{1}$, Mario DiDea, RT(N) ${ }^{2}$, France Fournier, CNMT $^{3}$, Daniel Tempesta, \\ CNMT, RT(N)(CT) ${ }^{4}$, Jessica Williams, CNMT, RT(N), FSNMMI-TS ${ }^{5}$, and Norman LaFrance ${ }^{3}$ \\ ${ }^{1}$ Hartford Healthcare Corporation, Hartford, Connecticut; ${ }^{2}$ Montefiore Medical Center, New York, New York; ${ }^{3}$ Jubilant DraxImage, \\ Inc., Kirkland, Quebec, Canada; ${ }^{4}$ Beth Israel Deaconess Medical Center, Boston, Massachusetts; and ${ }^{5}$ Hospital of the University of \\ Pennsylvania, Philadelphia, Pennsylvania
}

\begin{abstract}
CE credit: For CE credit, you can access the test for this article, as well as additional JNMT CE tests, online at https://www.snmmilearningcenter.org. Complete the test online no later than June 2021. Your online test will be scored immediately. You may make 3 attempts to pass the test and must answer $80 \%$ of the questions correctly to receive $1.0 \mathrm{CEH}$ (Continuing Education Hour) credit. SNMMI members will have their CEH credit added to their VOICE transcript automatically; nonmembers will be able to print out a CE certificate upon successfully completing the test. The online test is free to SNMMI members; nonmembers must pay $\$ 15.00$ by credit card when logging onto the website to take the test.
\end{abstract}

As the health care environment continues to change and morph into a system focusing on increased quality and evidencebased outcomes, nuclear medicine technologists must be reminded that they play a critical role in achieving high-quality, interpretable images used to drive patient care, treatment, and best possible outcomes. A survey performed by the Quality Committee of the Society of Nuclear Medicine and Molecular Imaging Technologist Section demonstrated that a clear knowledge gap exists among technologists regarding their understanding of quality, how it is measured, and how it should be achieved by all practicing technologists regardless of role and education level. Understanding of these areas within health care, in conjunction with the growing emphasis on evidencebased outcomes, quality measures, and patient satisfaction, will ultimately elevate the role of nuclear medicine technologists today and into the future. The nuclear medicine role now requires technologists to demonstrate patient assessment skills, practice safety procedures with regard to staff and patients, provide patient education and instruction, and provide physicians with information to assist with the interpretation and outcome of the study. In addition, the technologist must be able to evaluate images by performing technical analysis, knowing the demonstrated anatomy and pathophysiology, and assessing overall quality. Technologists must also be able to triage and understand the disease processes being evaluated and how nuclear medicine diagnostic studies may drive care and treatment. Therefore, it is imperative that nuclear medicine technologists understand their role in the achievement of a high-quality, interpretable study by applying quality principles and understanding and using imaging techniques beyond just basic protocols for every type of disease or system being imaged. This article focuses on quality considerations related to ventilation-perfusion imaging. It provides insight on appropriate imaging techniques and protocols, true imaging variants and tracer distributions versus artifacts that may result in a lower-

\footnotetext{
Received Mar. 10, 2018; revision accepted Apr. 17, 2018.

For correspondence or reprints contact: April Mann, Heart and Vascular Institute, Hartford Healthcare Corporation, 80 Seymour St., JB 722 Suite M, Hartford, CT 06102.

E-mail: april.mann@hhchealth.org

Published online May 3, 2018

COPYRIGHT (c) 2018 by the Society of Nuclear Medicine and Molecular Imaging.
}

quality or misinterpreted study, and the use of SPECT and SPECT/CT as an alternative providing a high-quality, interpretable study with better diagnostic accuracy and fewer nondiagnostic procedures than historical planar imaging.

Key Words: pulmonary V/Q imaging; SPECT; SPECT/CT

J Nucl Med Technol 2018; 46:89-95

DOI: 10.2967/jnmt.118.210948

\section{A} the health care environment continues to change and morph into a quality system focused on evidence-based outcomes, the nuclear medicine community continues to face external pressures, including scrutiny of patient radiation exposure, a continued perception of overutilization of testing, decreasing reimbursement, and fewer technologist jobs as a result of fewer studies being ordered and continued budget cuts. These changes in health care, coupled with additional external pressures, will play a significant role in molding the future of the field. Therefore, now more than ever, it is extremely important to acquire the imaging study right the first time. To remain successful in this ever-changing health care environment, it will be vitally important for technologists to understand their role in achieving a high-quality, interpretable study to drive positive patient care, treatment, and outcomes.

\section{QUALITY SYSTEMS IN HEALTH CARE}

\section{Background}

Quality systems focusing on evidence-based patient outcomes and satisfaction are being used by the Centers for Medicare and Medicaid for reimbursement and education, from competency to recertification. Some such measures for hospitals include tracking of the incidence of hospital-acquired infections or deep-vein thrombosis, heart failure readmission rates, and length of stay for many conditions. Additionally, several states have mandated reporting of specific measures, and private payers are using 
other, similar, quality initiatives as an efficient way to cut costs and control reimbursement.

Although the strong emphasis on quality systems still seems relatively new to many health care professionals, in 2001 the Institute of Medicine published a landmark report identifying major gaps in the American health care system $(1,2)$. This report urgently called for closure of these gaps by a redesign of the American health care system and provided overarching principles and specific direction for policy makers, health care leaders, clinicians, regulators, purchasers, and others health care providers (2). These principles also included 6 elements every health care system should encompass to achieve high-quality patient care: safety (avoiding injuries to patients from the care that is intended to help them (1)); effectiveness (providing services based on scientific knowledge to all who could benefit, and refraining from providing services to those not likely to benefit (1)); patientcenteredness (providing care that is respectful of and responsive to individual patient preferences, needs, and values, and ensuring that patient values guide all clinical decisions); timeliness (reducing waits and sometimes-harmful delays both for those who receive care and for those who give care (1)); efficiency (avoiding waste, including waste of equipment, supplies, ideas, and energy (1)); and equitability (providing care that does not vary in quality because of personal characteristics such as sex, ethnicity, geographic location, and socioeconomic status (1)).

In addition to these 6 areas of focus, the Institute of Medicine also defined core competencies for health care professionals related to patient quality and safety. These competencies require all involved in the care and treatment of patients to understand and provide patient-centered care, work in interdisciplinary teams, and employ evidencebased practice through application of quality improvement and use of informatics to drive change (1).

\section{Role of Nuclear Medicine Technologist}

In 2015, the Quality Committee of the Society of Nuclear Medicine and Molecular Imaging Technologist Section developed a survey to identify the level of knowledge about the above-mentioned concepts and principles of quality among nuclear medicine technologists (2). Created by the Quality Committee in collaboration with McKinley Advisors, this survey, which was administered from July 28 to August 18, 2016, was necessary to gain insight into how quality is viewed, measured, and learned in the nuclear medicine field. The Technologist Section partnered with the American Registry of Radiologic Technologists and the Nuclear Medicine Technology Certification Board to allow the survey to reach a broader audience. The survey was send to 27,989 certified or registered technologists and garnered 4,007 responses, for a $14.3 \%$ response rate. The results demonstrated a clear knowledge gap in the level of understanding regarding quality, how it is measured, and how it should be achieved among all levels of technologists despite role (staff to administrators) and education (asso- ciate through masters). In addition, the results showed the immediate need to develop education for technologists and create awareness about quality across the continuum of patient care and how it relates to imaging procedures. The full survey results and findings were published in the June 2017 edition of the Journal of Nuclear Medicine Technology (2).

One of the most important goals within the health care field is to understand and transition to evidence-based outcomes and quality measures to drive high-level performance. The nuclear medicine technologist of today must be able to demonstrate patient assessment skills, practice safety procedures with regard to staff and patients, provide patient education and instruction, and provide the physician with any information pertinent to the potential interpretation and outcome of a study. In addition, the technologist must be able to evaluate images by performing technical analysis, knowing the demonstrated anatomy, and assessing overall quality. Technologists must also be able to triage and understand the disease processes being evaluated and how nuclear medicine diagnostic studies may drive care and treatment. Therefore, it is imperative that nuclear medicine technologists understand their role in achieving a high-quality, interpretable study by applying quality principles and using imaging techniques beyond just basic protocols for every type of disease or system being imaged.

\section{QUALITY IN VENTILATION-PERFUSION (V/Q) IMAGING}

Nuclear medicine studies provide valuable functional and pathophysiologic information on many organ-specific areas, particularly V/Q imaging of the lungs. V/Q imaging for the detection and diagnosis of pulmonary embolism (PE) has been performed for over $50 \mathrm{y}$, offers a reliable alternative to other invasive and noninvasive procedures available, and has contributed to making nuclear medicine a viable and critically important option for patient management (3-5). When first offered, before the availability of ${ }^{99 \mathrm{~m}} \mathrm{Tc}$, studies were performed with ${ }^{131} \mathrm{I}$ on a rectilinear scanner and provided valuable information to the referring physician. Since that time, however, the field has seen many changes with the advent of newer technology, and CT pulmonary angiography (CTPA) has earned growing respect for the diagnosis of PE despite limitations such as contrast allergies, excessive absorbed radiation dose to the female breast, and risk of renal dysfunction or impairment from iodinated contrast administration $(6,7)$.

Of concern and requiring ongoing and further consideration is a randomized clinical trial demonstrating that $\mathrm{PE}$ is diagnosed in significantly more patients by CTPA than by V/Q because the former can detect clinically insignificant pulmonary perfusion defects. This overdiagnosis of PE poses a risk to patients due to unnecessary use of anticoagulant therapy for small emboli that are not likely to be clinically relevant (6). These data in the nonimaging medical literature further stated that even with this higher rate of diagnosis by CTPA, and the higher morbidity and mortality from increased anticoagulation therapy, the outcome for PE did not improve. 
For V/Q imaging to be reliable compared with CTPA, however, a high-quality, interpretable study must be achieved. The nuclear medicine technologist plays a key role in ensuring the quality of the study and should attend to all aspects of imaging before, during, and after the procedure: from initial patient presentation through image interpretation.

\section{Nonimaging Considerations}

With any imaging procedure, it is important to identify and address nonimaging considerations before performing the study. These include the timeliness of scheduling, the appropriateness of the procedure being performed, the creation of a positive experience for patients, and timely communication of results by appropriate personnel. An appropriate procedure is defined as one for which the expected health benefits exceed the expected health risks by a wide margin $(3,8)$. Further, when imaging is considered, appropriate-use criteria are defined as "criteria that are evidence-based (to the extent feasible) and assist professionals who order and furnish applicable imaging services to make the most appropriate treatment decisions for a specific clinical condition (8)." Nuclear medicine has a unique position in the health care benefit-risk paradigm. Because nearly all the diagnostic procedures have very low to negligible risk, the benefit-to-risk ratio is usually very much in favor of benefit.

\section{Clinical Indications}

A valuable role for technologists may be in helping to determine the appropriateness of a V/Q study by verifying the clinical indication and any other pertinent patient history before performing the procedure. The most common clinical indication for a V/Q study is acute or chronic PE. Less common indications include, but are not limited to, documentation of the resolution of $\mathrm{PE}$, quantification of differential function before pulmonary surgery (e.g., lung cancer), evaluation of lung transplants, evaluation of the effects of congenital heart or lung disease (e.g., cardiac shunts, pulmonary arterial stenosis, and arteriovenous fistula) and follow-up and monitoring of their treatment, confirmation of the presence of bronchopleural fistulae, evaluation of the effects of chronic pulmonary parenchymal disorders such as cystic fibrosis, evaluation of alveolar clearance or function (e.g., smoking history and inhalation or other environmental injury or exposure), and evaluation of the cause of pulmonary hypertension $(3,4,9,10)$. Medically, there are no absolute contraindications to be considered other than documented hypersensitivity reactions, which are rare. However, if a female patient is pregnant or nursing, she should be precautioned and counseled before the procedure, and the benefit to the mother must outweigh the slight radiation exposure to the fetus.

\section{Patient Preparation}

Care should be taken to prepare the patient before the procedure. A standard chest radiograph in the posterior-anterior and lateral positions is preferred and usually performed within $24 \mathrm{~h}$ of the V/Q study unless a more acute clinical change has occurred (9). A portable chest radiograph in the anterior-posterior position is acceptable if the patient's condition so merits or the patient cannot tolerate a standard chest radiograph. A chest CT scan, if available, can be used as a substitute for a standard chest radiograph. A previous chest radiograph performed a few days before the V/Q study may be acceptable if the patient has had no change in signs, symptoms, or clinical situation (9). To ensure patient cooperation, the technologist should explain the procedure in detail and address any questions or concerns. A demonstration of what to expect during the procedure may be appropriate for certain patients and should be done before the patient enters the imaging room, preferably immediately after the technologist greets the patient. In addition, all women of childbearing age should be questioned about pregnancy before receiving any radiopharmaceutical.

\section{Imaging Sequence}

A V/Q study typically begins with the ventilation study (9). Alternatively, the perfusion study may be performed first and the ventilation study eliminated if perfusion is normal or matches the previous chest radiography findings. This approach may be preferred in pregnant women, for example. A disadvantage to performing the perfusion study first is that the ventilation images may show radioisotope activity in the background. Also, a timely decision must be made afterward on whether the ventilation study is warranted (9), requiring the availability of a physician for immediate interpretation.

\section{Ventilation Imaging}

Ventilation imaging may be performed using either a radioactive gas or a radioaerosol. Either is acceptable for a planar acquisition, whereas a radioaerosol, by allowing multiple planar views, is required for a SPECT acquisition. As in all imaging procedures, the technologist should ensure that quality control testing was completed and that system performance is acceptable before performing the study.

Radioactive Gas. Radioactive gas ventilation imaging is generally performed using ${ }^{133} \mathrm{Xe}$, which is supplied in unitdose vials and administered by continuous inhalation. Adults receive $185-740 \mathrm{MBq}(5-20 \mathrm{mCi})$, and children receive 11.1 $\mathrm{MBq} / \mathrm{kg}(0.3 \mathrm{mCi} / \mathrm{kg})$ with a minimum of $111 \mathrm{MBq}(3.0$ $\mathrm{mCi}$ ) (9). The imaging room should be appropriately exhausted, including being a negative-pressure area when required by regulations. These regulations vary by geographic location, and it is important for technologists to know the requirements in their specific state or locality. Safe handling procedures for radioactive gas must be followed at all times and comply with all local, state, or federal regulations.

The gas should be administered through a facemask, or through a mouthpiece with the nose clipped shut, using a gas delivery system. Whenever possible, images should be acquired with the patient upright for best visualization of lung capacity. For some patients, a demonstration of proper breathing technique may be warranted to ensure adequate lung uptake. Single-breath images, wash-in (equilibrium) images, and washout images should be obtained in the posterior view. 


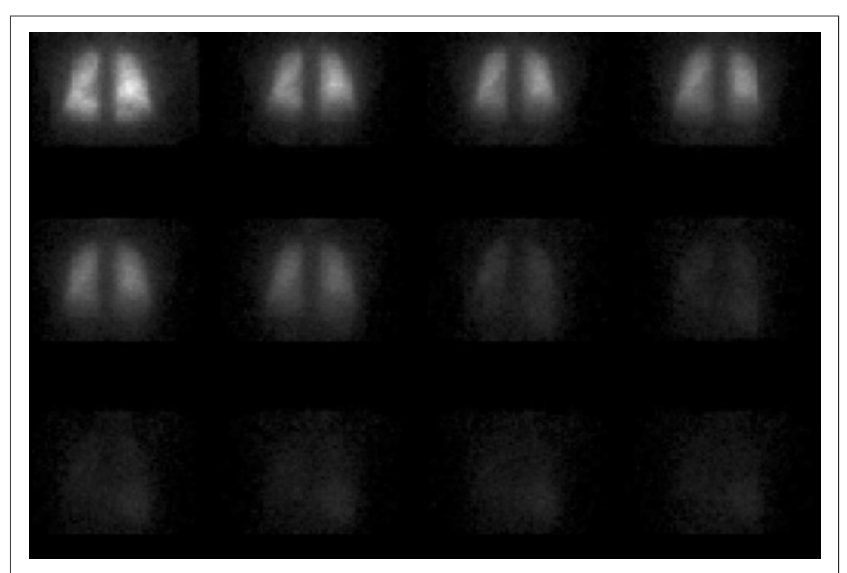

FIGURE 1. Normal ${ }^{133} \mathrm{Xe}$ gas ventilation findings.

Equilibrium images should be acquired for 3-4 min, as tolerated (15-60 s/image) (Fig. 1) (9). When possible, posterior oblique washout images should be obtained to add ventilation information for optimal evaluation of V/Q match or mismatch. If perfusion imaging is performed first, a ${ }^{99 \mathrm{~m}} \mathrm{Tc}$ background image should be obtained using the ${ }^{133} \mathrm{Xe}$ window. The patient should be positioned using the view best visualizing the perfusion defect. Gas ventilation has several advantages: singlebreath, wash-in (equilibrium), and washout images can be obtained; characterization of ventilation may be more complete; sensitivity for evaluation of obstructive airway disease may be higher; and, with ${ }^{133} \mathrm{Xe}$, the best physiologic ventilation information may be obtained (9).

Radioaerosol. For 3 decades, radioaerosol ventilation imaging has used ${ }^{99 \mathrm{~m}} \mathrm{Tc}$-diethylenetriamine pentaacetic acid (DTPA) almost exclusively - the only radioisotope approved for this use $(9,11)$. ${ }^{99 \mathrm{~m} T c}$-sulfur colloid has also been considered for use by some nuclear medicine physicians and is believed to have a slower lung clearance (9). The standard activity of ${ }^{99 \mathrm{~m}} \mathrm{Tc}$-DTPA dispensed in the nebulizer is $925-1,295 \mathrm{MBq}(25-35 \mathrm{mCi})$, of which the patient's lungs usually receive about $18.5-37 \mathrm{MBq}(0.5-1.0$ $\mathrm{mCi})$, or $2 \%-10 \%$ of the dose. Ventilation imaging using a radioaerosol should be performed before perfusion imaging because of dose-range variability limitations. The count rate of the second study using a single radioisotope with the same energy (i.e., ${ }^{99 \mathrm{~m}} \mathrm{Tc}$ ) should be $3-4$ times the rate of the first study $(4,9)$. This higher rate is difficult to achieve with a radioaerosol dose. To ensure a high-quality image, the count rate should be assessed on the posterior image. If the count rate is too low, the technologist should first check for adequate nebulizer reservoir activity and loading. If required for an acceptable-quality study, the patient should breathe through the nebulizer for an additional few minutes.

The radioaerosol must be administered through a welldesigned, high-quality, approved nebulizer, of which several types are available. It is a critical responsibility of the technologist to fully understand the delivery procedure for the specific nebulizer system being used and to perform the required maintenance and cleaning as directed by the manufacturer. The radioaerosol should be delivered to the patient through a mouthpiece with the nose clipped shut; alternatively, a facemask may be used if the patient cannot breathe appropriately through the mouthpiece. It is recommended that the technique be demonstrated for all patients to ensure proper distribution in the alveoli.

Whenever possible, images should be acquired with the patient upright, with multiple views acquired (standard anterior, posterior, left and right lateral, left and right anterior oblique, and left and right posterior oblique) for at least 300,000 counts per image (Fig. 2) (9). If a patient cannot be imaged upright, the arms and other possible sources of attenuation should be kept out of the field of view. Radioaerosol ventilation has several advantages: images may be obtained in multiple projections; SPECT imaging may be performed (and is recommended by some experts); images may be obtained at the bedside if necessary, since a negativepressure room is not required; ${ }^{99 \mathrm{~m}} \mathrm{Tc}-\mathrm{DTPA}$ may be prepared on an as-needed basis as opposed to having ${ }^{133} \mathrm{Xe}$ vials always available, eliminating potential wastage and decreasing costs; and ${ }^{99 \mathrm{~m}} \mathrm{Tc}-\mathrm{DTPA}$ may provide unique information on alveolar-clearance airway disease and other pulmonary diseases because of the consistent and reproducible ${ }^{99 \mathrm{mTc}-}$ DTPA aerosol size generated at room temperature.

\section{Perfusion Imaging}

Perfusion imaging is performed using ${ }^{99 \mathrm{~m}} \mathrm{Tc}$-macroaggregated albumin (MAA). The standard administered dose in adults is $37-148 \mathrm{MBq}(1-4 \mathrm{mCi})$. In children, the dose is $11.1 \mathrm{MBq} / \mathrm{kg}(0.03 \mathrm{mCi} / \mathrm{kg})$ (minimum, $14.8 \mathrm{MBq}[0.4$ $\mathrm{mCi}]$ ) when ventilation images are not obtained and 2.59 $\mathrm{MBq} / \mathrm{kg}(0.07 \mathrm{mCi} / \mathrm{kg})$ when they are $(9,12)$. The particle number per dose should be 200,000-700,000, with at least 350,000 being recommended $(9,12)$. The ${ }^{99 m}$ Tc-MAA package insert specifically recommends caution if the particle number is below 200,000, and fewer than 350,000 particles should be considered only in patients with pulmonary hypertension or right-to-left shunting, in infants and children, and in patients with a single lung transplant (12).

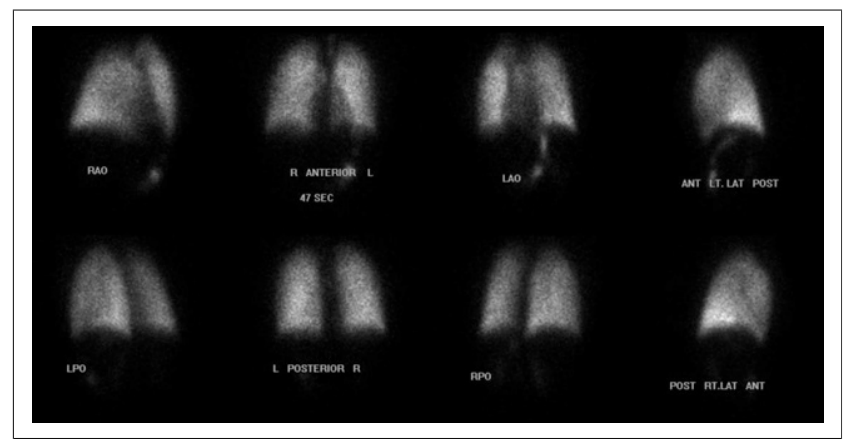

FIGURE 2. Normal ${ }^{99 m}$ Tc-DTPA radioaerosol findings. ANT = anterior; LAO = left anterior oblique; LAT = lateral; LPO = left posterior oblique; POST = posterior; RAO = right anterior oblique; RPO = right posterior oblique. 
Dose volume may vary from 0.2 to $1.4 \mathrm{~mL}$, depending on vial reconstitution, and the vial should be gently agitated immediately before dose withdrawal $(8,11)$. Additionally, the syringe should be gently inverted several times before injection, because labeled ${ }^{99 \mathrm{~m}}$ Tc-MAA particles will settle over time $(9,12)$.

The patient should be instructed to cough and take several deep breaths before intravenous administration of the ${ }^{99 m}$ Tc-MAA, which should be injected slowly over 3-5 respiratory cycles with the patient supine (9). The radiotracer should not be injected into a distal (Swan-Ganz) catheter or any indwelling line or port containing a filter because the particles will be trapped and not reach the patient's pulmonary artery vasculature. If it is necessary to make the injection through an indwelling intravenous line, the line should be flushed generously to mitigate loss of dose through adherence to the tubing. Perfusion images should be acquired with the patient upright to increase pulmonary volume and minimize diaphragmatic motion. If planar imaging is performed, views should be acquired in the anterior, posterior, left and right anterior oblique, left and right posterior oblique, and left and right lateral positions for 300,000-500,000 counts (Fig. 3) (9).

\section{Pitfalls Affecting Quality}

In any imaging study, there are several factors affecting overall image quality that may ultimately have an impact on interpretation and patient outcome. These factors include patient preparation, overall system performance, patient positioning, dose, imaging technique, acquisition protocol, and acquisition parameters. If not adequate, all these factors may have a negative impact on patient outcome. The nuclear medicine technologist is a critical first line of defense against poor image quality. The interpretation can only be as reliable as the quality of the images achieved. Therefore, it is the primary responsibility of the technologist to ensure that a high-quality, interpretable image is acquired for every study. V/Q imaging has several technical considerations that, if not attended to, may result in a suboptimal acquisition that risks poor interpretation.

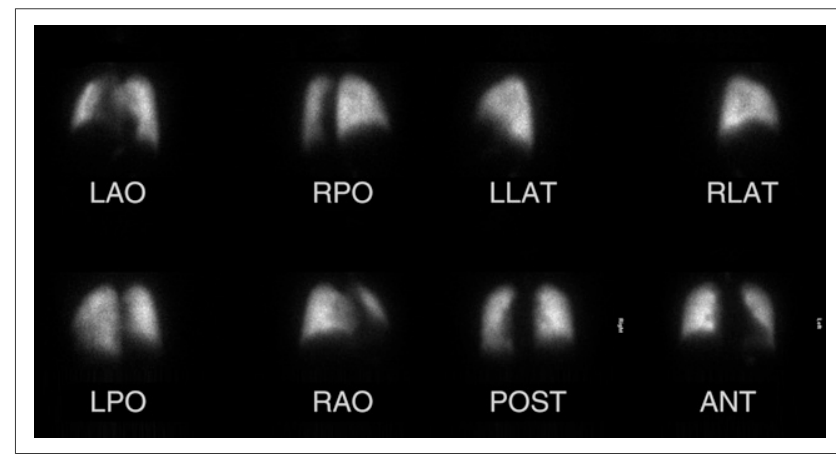

FIGURE 3. Normal ${ }^{99 m}$ Tc-MAA lung perfusion findings. ANT = anterior; LAO = left anterior oblique; LLAT = left lateral; LPO = left posterior oblique; POST = posterior; RAO = right anterior oblique; RLAT = right lateral; RPO = right posterior oblique.

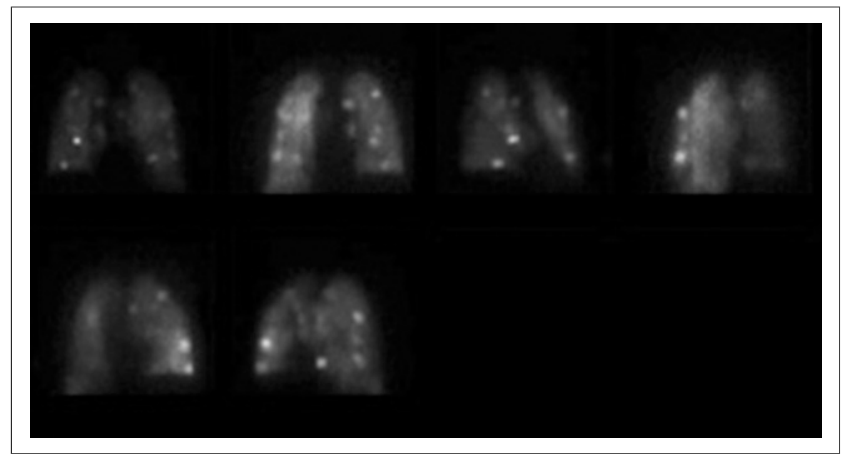

FIGURE 4. Hot spots on 99mTc-MAA study due to blood clotting in syringe.

The most common types of artifacts on perfusion images are due to inappropriate ${ }^{99 m}$ Tc-MAA injection techniques or poor kit reconstitution. This type of artifact can include hot spots on the perfusion images due to blood clotting in the syringe during injection or, if the injection is through an indwelling catheter, lack of sufficient flushing (Fig. 4). Another artifact of this type is an inadequate, inhomogeneous distribution of activity due to a low particle count in the administered dose or, if the patient is injected while upright, more counts in the base of the lungs than elsewhere (Figs. 5 and 6). All such artifacts may be prevented by following appropriate techniques for patient preparation, kit reconstitution, patient positioning, and injection.

On ventilation images, artifacts may arise from poor breathing by the patient. Such artifacts include activity visualized in the esophagus and mouth or clumping of the radioaerosol (Fig. 7). These types of artifacts may be prevented by ensuring that the patient understands the procedure and is given adequate instructions, coaching, and demonstrations, if necessary, before undergoing the study. If excessive activity is seen in the mouth or esophagus, the lungs should be positioned at the top of the field of view. Doing so may eliminate this activity from the field of view

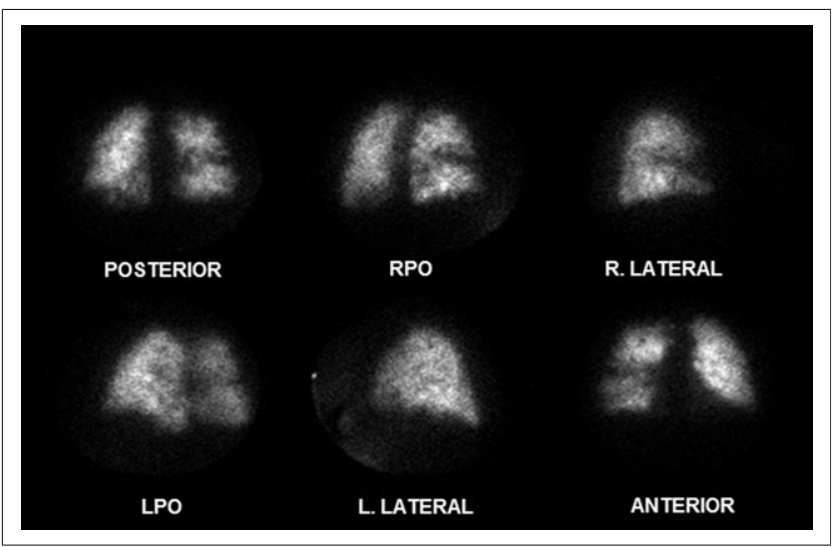

FIGURE 5. Inhomogeneous activity on 99mTc-MAA perfusion study due to low particle count. LPO = left posterior oblique; $\mathrm{RPO}=$ right posterior oblique. 


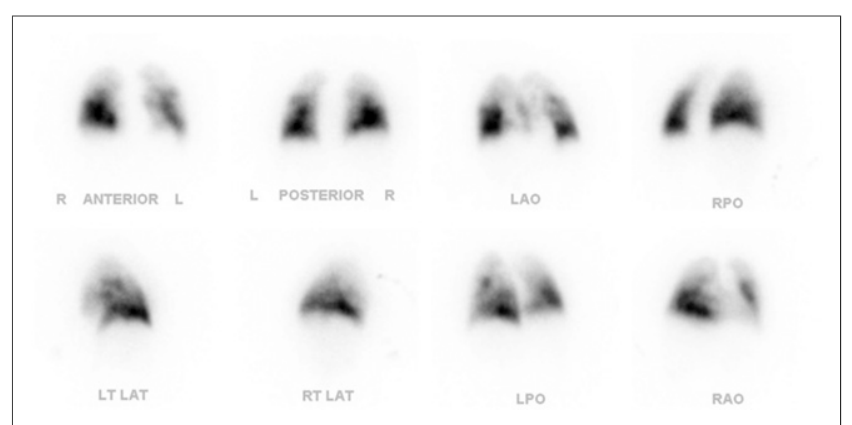

FIGURE 6. Inhomogeneous activity on 99mTc-MAA perfusion study due to patient being injected while upright instead of supine. LAO = left anterior oblique; LPO = left posterior oblique; $\mathrm{RAO}=$ right anterior oblique; RPO $=$ right posterior oblique.

and avoid falsely high count-rates in the images, which may affect the ability to achieve 3-4 times the count of the perfusion study. If the patient can cooperate, rinsing the mouth with a small volume of water and expectorating can eliminate mouth and esophageal activity. Although mouth or esophageal radioaerosol activity is usually due to poor nebulizer technique, airway visualization often represents pulmonary airway pathophysiology and may deserve further evaluation if not already known about.

Additionally, a patient who cannot be upright for the ventilation study should be positioned the same as in the perfusion study. Placement of the arms or other attenuation sources within the field of view may create a false-positive mismatch pattern related not to a clinical condition but to poor positioning. Occasionally, artifacts may also occur because the patient's clothing has become contaminated by the radioisotope. This issue simply requires changing of the clothing or gown to remove the contamination from the field of view.

Finally, it is important that the ventilation study and the perfusion study be performed within a short time of each other $(9,10)$. Allowing too much time between them may affect interpretation because of interim changes in the patient's pathophysiology, signs, symptoms, and clinical presentation. Patients undergoing V/Q studies may be critically compromised and should never be left unattended. Serious PE involvement may be present even when there are no outward signs or symptoms such as chest pain, shortness of breath, hemoptysis, or other high-risk presentations and no history of deep-vein thrombosis, malignancy, or recent long trips (9). In addition, tachycardia is a classic symptom of PE but, combined with low $\mathrm{O}_{2}$ saturation and distension of neck vessels, may represent either cardiac tamponade or PE. A technologist who is aware of and vigilant about the artifacts or complications that may arise during a V/Q study will produce a higher-quality study and an overall more positive patient experience.

\section{Image Interpretation and Reporting}

Although image interpretation and reporting are not the primary professional responsibility of technologists, they should understand what adequate interpretation requires and communicate any observations to the interpreting physician. They should also understand how to produce images of sufficient quality for accurate interpretation, allowing the referring physician to make a proper clinical decision. Most importantly, the report should include clinical findings and an assessment of image quality (9), as optimal-quality images should always lead to a quality interpretation. The report may also assess the postimaging probability of pulmonary PE versus the preimaging probability (9), although some experts are now recommending 3 diagnostic categories: PE present, PE absent, and nondiagnostic or uninterpretable study (9). This approach may correct the negative V/Q bias precipitated by the Prospective Investigation of Pulmonary Embolism Diagnosis Study (PIOPED) mandating pre- and posttest probability criteria for V/Q studies versus the tertiary interpretation criteria allowed for CTPA. Still other experts suggest that more accurate categorization provides more information to referring physicians. The discussion of PIOPED II by Sostman et al. provides an in-depth explanation of image interpretation $(13,14)$.

Some experts believe that PIOPED created unintended consequences and that probability-based interpretation and the widely variable probability language has confused radiologists and diminished their confidence in V/Q studies. This diminished confidence, combined with more recent advances in technology, has led to favoring of CTPA over functional (V/Q) imaging (6). However, the detection of clinically insignificant clots (by CTPA) could unnecessarily expose patients to the risks of anticoagulant therapy. Just as there is sometimes a rationale for favoring V/Q studies over CTPA, such as in high-risk patients (e.g., those with

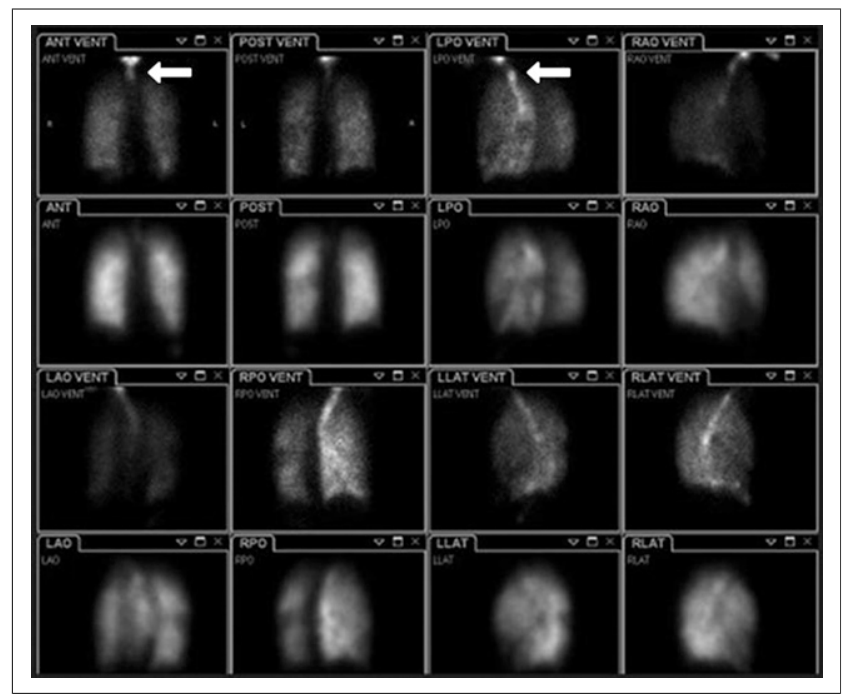

FIGURE 7. Activity (arrows) visualized in esophagus and mouth during 99mTc-DTPA ventilation study. ANT = anterior; LAO = left anterior oblique; LLAT = left lateral; LPO = left posterior oblique; $\mathrm{POST}=$ posterior; $\mathrm{RAO}=$ right anterior oblique; RLAT = right lateral; RPO = right posterior oblique; VENT $=$ ventral. 
diabetes mellitus, iodinated contrast sensitivity, renal dysfunction or failure, or higher absorbed-radiation exposure), there are also situations in which CTPA may be preferred, such as when there is a high likelihood of non-PE pulmonary pathology or when there are significantly or diffusely abnormal findings on chest radiography (6).

\section{SPECT and SPECT/CT Studies}

The field of nuclear medicine technology, as well as the V/Q study, has evolved greatly over the past 50 y (15). Despite this evolution, V/Q imaging in most facilities is still being performed using technology and techniques similar to those of $25 \mathrm{y}$ ago, and PIOPED - the landmark accuracy study for V/Q imaging-uses mostly planar data (13-15). However, some investigators believe the technology and agents for V/Q imaging have evolved rapidly in recent years (15). Le Roux et al. and others have suggested that this evolution in technology has improved the diagnostic performance of SPECT V/Q imaging, with a decrease in the number of nondiagnostic procedures. Furthermore, SPECT has replaced planar imaging for the diagnosis of PE in Australia, Canada, and France $(8,15,16)$. Several studies have shown that in SPECT/CT V/Q imaging, not only is the specificity and overall diagnostic accuracy of the V/Q study improved, but the ventilation aerosol SPECT/CT study also adds practical clinical information when combined with a perfusion SPECT study $(9,15,16)$. Some investigators further suggest that SPECT/CT should be considered for the diagnosis of PE because the low-dose CT component may provide attenuation information and better anatomic information than a chest radiograph alone $(15,16)$.

As the technology evolves and SPECT and SPECT/CT come into more common use for V/Q imaging, caution will be needed to ensure that both technologists and physicians are appropriately trained to perform the SPECT acquisition, including how to recognize new normal variants and artifacts differing from those of planar imaging and how the improved sensitivity and specificity may affect interpretation and outcomes. Like the concerns about CTPA, there are concerns that SPECT/CT V/Q imaging may visualize clinically insignificant clots, resulting in more patients being diagnosed with $\mathrm{PE}$ and receiving potentially unnecessary anticoagulant therapy (6). In this regard, further study and evaluation are needed to determine the potential extent and impact of the use of SPECT and SPECT/ CT for V/Q imaging.

\section{CONCLUSION}

There are many steps the nuclear medicine technologist can take to optimize a V/Q study, such as following the required imaging standards, distinguishing abnormal distribution patterns from artifacts, doing the right and safe thing for patients, providing a positive patient experience, and minimizing unnecessary scans. V/Q imaging is a valuable noninvasive procedure for diagnosing cardiac or pulmonary disorders such as PE and is endorsed by the American College of Emergency Physicians. Use of appropriate technique is needed to optimize image quality and achieve accurate, reliable results and will become more important as V/Q imaging moves toward SPECT and SPECT/CT. Regardless of the modality, image interpretation is only as good as the images obtained, and a successful outcome relates directly to the quality of the interpretation. The nuclear medicine technologist has a critical role in achieving a high-quality, interpretable study that drives positive patient care, treatment, and outcomes.

\section{DISCLOSURE}

No potential conflict of interest relevant to this article was reported.

\section{REFERENCES}

1. Institute of Medicine. Crossing the Quality Chasm: A New Health System for the 21st Century. Washington, DC: The National Academies Press; 2001.

2. Mann A, Farrell MB, Williams J, Basso D. Nuclear medicine technologists' perception and current assessment of quality: a Society of Nuclear Medicine and Molecular Imaging Technologist Section survey. J Nucl Med Technol. 2017;45:67-74.

3. Waxman AD, Bajc M, Brown M, et al. Appropriate use criteria for ventilationperfusion imaging in pulmonary embolism: summary and excerpts. J Nucl Med. 2017;58(5):13N-15N.

4. Hur S, Bauer A, McMillan N, Krupinski EA, Kuo PH. Optimizing the ventilation-perfusion lung scan for image quality and radiation exposure. J Nucl Med Technol. 2014;42:51-54.

5. Five things patients and providers should question. Choosing Wisely website. http://www.choosingwisely.org/wp-content/uploads/2015/01/Choosing-WiselyRecommendations.pdf. Published November 15, 2017. Accessed April 24, 2018.

6. Anderson DR, Kahn SR, Rodger MA, et al. Computed tomographic pulmonary angiography vs. ventilation-perfusion lung scanning in patients with suspected pulmonary embolism: a randomized controlled trial. JAMA. 2007;298:27432753.

7. Appropriate use criteria program. CMS.gov website. https://www.cms.gov/Medicare/Quality-Initiatives-Patient-Assessment-Instruments/Appropriate-Use-CriteriaProgram/index.html. Updated November 29, 2017. Accessed April 24, 2018.

8. Brenner DJ, Hall EJ. Computed tomography: an increased source of radiation exposure. N Engl J Med. 2007;357:2277-2284.

9. Parker JA, Coleman RE, Grady E, et al. SNM practice guidelines for lung scintigraphy 4.0. J Nucl Med Technol. 2012;40:57-65.

10. ACR-SNM-SPR practice guidelines for the performance of pulmonary scintigraphy in adults and children. SNMMI website. http://snmmi.files.cms-plus.com/ docs/Pulmonary_Scintigraphy_1382732040599_8.pdf. Published 1995. Last revised 2009. Accessed April 24, 2018.

11. DraxImage DTPA [package insert]. Kirkland, Quebec, Canada: Jubilant DraxImage, Inc.; 2018.

12. DraxImage MAA [package insert]. Kirkland, Quebec, Canada: Jubilant DraxImage, Inc.; 2018.

13. Sostman HD, Stein PD, Gottschalk A, et al. Acute pulmonary embolism: sensitivity and specificity of ventilation-perfusion scintigraphy in PIOPED II study. Radiology. 2008;246:941-946.

14. Sostman HD, Miniati M, Gottschalk A, et al. Sensitivity and specificity of perfusion scintigraphy combined with chest radiography for acute pulmonary embolism in PIOPED II. J Nucl Med. 2008;49:1741-1748.

15. Le Roux PY, Pelletier-Galarneau M, De Laroche R, et al. Pulmonary scintigraphy for the diagnosis of acute pulmonary embolism: a survey of current practice in Australia, Canada and France. J Nucl Med. 2015;56:1212-1217.

16. Roach PJ, Schembri GP, Baily DL. V/Q scanning using SPECT and SPECT/CT. J Nucl Med. 2013;54:1588-1596. 